\title{
Achieving Fairness in Lossy 802.11e Wireless Multi-Hop Mesh Networks
}

\author{
Qizhi Cao, Tianji Li Member, IEEE, and Douglas Leith, Member, IEEE,
}

\begin{abstract}
We consider achieving max-min fairness in 802.11e based multi-hop wireless networks. We propose an approach which makes use of the TXOP mechanism in combination with an automatic contention window size tuning algorithm based on channel state sensing. Simulation results show that the proposed approach can provide a good approximation to per-flow max-min fairness and that this is achieved regardless of the active number of flows and when the channel is noisy.
\end{abstract}

Index Terms-Medium access control (MAC), IEEE 802.11, IEEE 802.11e

\section{INTRODUCTION}

802.11 technology is becoming increasing pervasive as the last-hop both in office environments and in the home. Looking ahead, the next step is likely to be towards greater use of multiple wireless hops. This not only includes the use of wireless for broadband backhaul infill, but also provision of municipal and rural wireless multi-hop networks. Within the home, moves towards Wifi-enabled multimedia distribution also lead almost inevitably to consideration of multiple wireless hops.

While there exists a considerable body of literature relating to 802.11 wireless multi-hop networks, much of this focusses on issues related to interference and routing which are wellknown difficult problems in single channel 802.11 networks. For example, it has been observed that due to hidden terminal effects end-to-end traffic over more than around 3 hops tends to achieve rather limited throughput [9]. Recently, there has been great interest in the use of multi-radio multi-channel networks. This reflects technology road-maps, and also the fact that multi-radio architectures combined with appropriate channel allocations potentially offer practically effective solutions to interference management, see for example [19], [20], [26], [3], [16] [14] and references therein.

In our previous work [15], we demonstrated that gross unfairness can exist among competing flows in an 802.11 mesh network. In this paper, extend this previous work in a number of directions. First, we demonstrate that in general the TXOPonly mechanism proposed in [15] is not sufficient to achieve max-min fairness. The reason for this is that MAC layer contention can unfairly penalize flows that traverse multiple hops. Second, we propose extending the TXOP approach to include autotuning of the 802.11 contention window and demonstrate via simulations that this combined approach succeeds in achieving max-min fairness across a wide range of network conditions. Third, we extend consideration to links

This work is supported by Science Foundation Ireland Grant 03/IN3/I396 and Irish Research Council for Science, Engineering and Technology.

Email: \{cao.qizhi, tianji.li, doug.leith\}@nuim.ie). with channel noise losses. Recent theoretical results in [23] establish that the max-min fair allocation varies smoothly as the level of losses is increased and we confirm similar behaviour in our simulations. Thus, provided the noise losses are not too high the proposed algorithm continues to achieve close to max-min fairness.

\section{BACKGROUND}

The 802.11e MAC protocol [1] extends the standard 802.11 DCF (Distributed Coordinated Function) contention mechanism by allowing the adjustment of MAC parameters that were previously fixed. With 802.11 , on detecting the wireless medium to be idle for a period DIFS, each station initializes a counter to a random number selected uniformly from the interval $[0, \mathrm{CW}-1]$ where $\mathrm{CW}$ stands for contention window. Time is slotted and this counter is decremented each slot that the medium is idle. An important feature is that the countdown halts when the medium becomes busy and only resumes after the medium is idle again for a period DIFS. On the counter reaching zero, the station transmits a packet. If a collision occurs (two or more stations transmit simultaneously), $\mathrm{CW}$ is doubled and the process repeated. On a successful transmission, $\mathrm{CW}$ is reset to the value $C W_{\min }$ and a new countdown starts for the next packet. The 802.11e MAC enables the values of DIFS (called $A I F S$ in 802.11e) and $C W_{\min }$ to be set on a per class basis for each station. Four separate classes at each station is specified in 802.11e. Packets from each class are put into a separate queue. Different parameters including AIFS, TXOP, $C W_{\min }, C W_{\max }$ can be assigned to each class/queue so that differentiations can be realised.

The TXOP mechanism specifies a duration during which a station can keep transmitting without releasing the channel once it wins a transmission opportunity. In order not to release the channel, a SIFS interval is inserted between each packetACK pair (Fig. 1). A successful transmission round consists of multiple packets and ACKs. By adjusting this time, the number of packets that may be transmitted by a station at each transmission opportunity can be controlled. A salient feature of the TXOP operation is that, if a large TXOP is assigned and there are not enough packets to be transmitted, the TXOP period is ended immediately to avoid wasting bandwidth.

\section{PREVIOUS WORK}

Most previous work in multi-hop networks has focussed on issues such as hidden terminals and interference (e.g., [19], [20], [26], [3], [16]). MAC-related unfairness has been studied in the context of single-hop 802.11 WLANs, e.g., see 


$$
\text { Idle }
$$

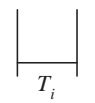

Successful

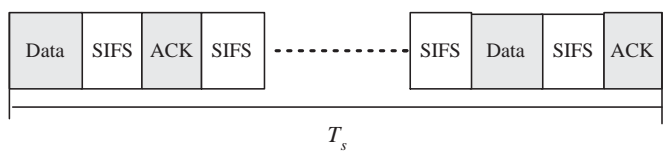

Collision

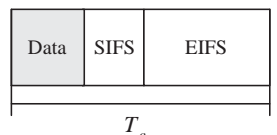

Fig. 1. Real slot durations in the 802.11e TXOP mechanism.

[13] [4] and references therein. However, fairness in multihop networks has received limited attention. In single-channel multi-hop networks, [9] illustrates that unfairness exists in parking lot deployments, and a congestion control algorithm is proposed to mitigate unfairness in [21]. The unfairness issue in [9] and [21] is caused by hidden terminals and interference. There has been even less work regarding the use of the TXOP mechanism. In [25], the authors evaluate the use of TXOP for stations with different physical rates.

\section{A. The TXOP Only Mechanism}

To the best of our knowledge, there exists no other prior work on enforcing/restoring per-flow fairness using 802.11e's TXOP in multi-hop networks besides our early work [15], [2]. This work proposes the following TXOP approach. At a transmission opportunity let the number of flows with packets queued at mesh point $M P_{i}$ on channel $l$ be $n$. Now use the TXOP duration $K_{l, i}=n$ packets plus a modified queuing discipline that serves one packet per flow at each transmission opportunity.

\section{B. A Counter Example}

The approach in [15] ignores the effect of packet losses on fairness. In networks with packet loss (which seems likely to be the normal situation) this can lead to unfairness. We illustrate this by an example. Consider the two-hop network shown in Fig. 2 in which user stations are marked by shadowed triangles, and mesh points (MPs) by circles. $M P_{1}$ is the station that relays traffic for user stations. $M P_{1}$ acts as a gateway between the wireless multi-hop network and the wired network. $M P_{0}$ has two radios that use channels in such a way that the channel in each hop is orthogonal to those in neighboring hops thereby avoiding interference between transmissions on different hops. Hence there are no hidden terminal effects. We assume that the set of routes from sources to destination are already obtained by all of the stations in the network. There are altogether 21 TCP flows in the example: 10 one-hop flows in each hop and 1 end-to-end flow (Flow 10) traversing two hops. In this topology, we expect that each flow should have the same throughput if the resulting allocation is fair. Using the TXOP only mechanism proposed in [15] however, we obtain the allocation shown in Fig. 3(a) using the parameters in Table I. As we can see the flow traversing two hops achieves a much lower throughput than the other flows. In this example, the number of active stations in each hop is relatively large (12 in each hop) and so the level of channel contention is sufficiently high to induce packet loss. The two-hop flow experiences a higher level of losses that the other flows since it must traverse two lossy hops rather than only one and it is this asymmetry that induces the observed unfairness.

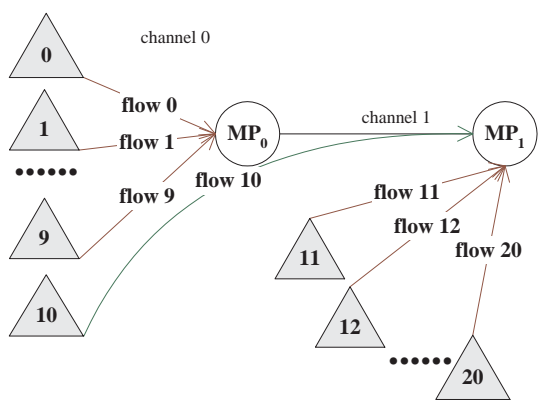

Fig. 2. Topology

\begin{tabular}{|l|l|}
\hline$T_{S I F S}(\mu s)$ & 10 \\
\hline Idle slot duration $(\sigma)(\mu s)$ & 20 \\
\hline$T_{D I F S}(\mu s)$ & 50 \\
\hline$C W_{\min }$ & 31 \\
\hline$C W_{\max }$ & 1023 \\
\hline Packet size (bytes) & 1000 \\
\hline PLCP rate (Mbps) & 1 \\
\hline
\end{tabular}

TABLE I

MAC AND PHY PARAMETERS USED IN SIMULATIONS.

\section{ACHIEVING FAIRnESS IN LOSSY NETWORKS}

The rationale behind the basic TXOP-only approach is as follows. Provided the buffering at wireless stations is sufficiently large, at a wireless hop we have that bottlenecked stations (where the mean arrival rate exceeds the mean service rate) are saturated and so achieve the same mean goodput (see for example the analysis in [17]). For other stations the mean goodput is equal to the mean arrival rate. The TXOP allocation proposed in [15] essentially creates a virtual station corresponding to each flow. We then have that backlogged flows at the same hop receive the same mean goodput, while for other flows the mean goodput equals to mean arrival rate. Now the TCP congestion control algorithm adjusts the offered load to ensure that every flow is bottlenecked and, when there are no packet losses, a flow can only be backlogged at a single hop. It therefore follows immediately that we have the conditions for a max-min fair allocation [12].

However, this argument neglects the impact of packet losses whereas in real networks losses are, of course, a common feature. Losses can occur, for example, due to repeated MAC 


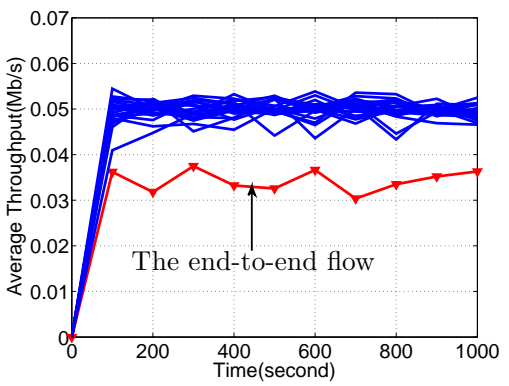

(a) TXOP-only approach from [15]



(b) With CW increased

Fig. 3. Impact of excessive MAC layer contention on fairness. The topology is shown in Fig. 3 and there are 10 local flows at hop 2 , retry limit 11 . In Fig.3(b) $C W_{\min }=1023$ and $C W_{\max }=1023$

collisions when a link is heavily contended, and due to channel noise. Recent theoretical results in [23] establish that the maxmin fair allocation varies smoothly as the level of losses is increased. Thus when the loss rate is low it will have only a small impact on fairness and we expect the TXOP-only approach in [15] to be effective. However, when loss rate is higher a new approach is needed, as illustrated by the counterexample above. In this section we focus on managing MAC layer contention related losses. Noise related losses are then considered later.

When the the level of MAC layer contention related losses on an 802.11 hop is too high it can be reduced by increasing the station $C W_{\min }$ values. For example, in Fig. 3(b), we increase $C W_{\min }$ from 31 to 1023 and observe that in the previous counter-example fairness is recovered as expected. The key difficulty of achieving this type of fairness in real networks lies in the fact that the channel capacity, channel state and the number of active stations and their traffic load are time-varying, difficult to predict and difficult to measure. The information required to determine a proper setting of contention window size is not available at any one station and so a decentralized $C W_{\min }$ tuning approach is fundamentally necessary. In this paper, we propose a practical decentralized approach that makes use of the channel-state sensing ability at each station. We then show that by combining this with the TXOP mechanism of [15], fairness can be consistently achieved, regardless of whether a link is lightly or heavily loaded.

\section{A. Regulating MAC contention}

We divide time into MAC slots, similar to those used in analytic models such as [17]. a MAC slot can be either (i) an idle slot where no station transmits, (ii) a successful slot where a single station transmits or (iii) a collision slot where one or more stations transmit. Letting $\tau_{i}$ denote the probability that a station attempts a transmission in a slot, the probability that a slot is idle is given by

$$
P_{i d l e, h}=\prod_{j \in N_{h}}\left(1-\tau_{j}\right)
$$

where $N_{h}$ is the set of stations that contend for access at the hop $h$ of interest. Intuitively, by maintaining $P_{i d l e, h}$ sufficiently large we expect that the channel is more likely to be clear when stations attempt a transmissions and so the collision rate is low.

More formally, we have that

$$
P_{i d l e, h}=\prod_{j \in N_{h}}\left(1-\tau_{j}\right) \geq P_{0}
$$

for some threshold $0<P_{0}<1$ implies that

$$
\tau_{i} \leq 1-P_{0}
$$

The probability that a transmission by station $i$ collides with a transmission by another station is

$$
P_{\text {coll }, i}=\tau_{i}\left(1-\prod_{j \in N_{h}, j \neq i}\left(1-\tau_{j}\right)\right)
$$

which can be rewritten as

$$
P_{\text {coll }, i}=\tau_{i}\left(1-\frac{P_{i d l e, h}}{1-\tau_{i}}\right)
$$

and so constraint (1) ensures that

$$
P_{\text {coll }, i} \leq 1-P_{0}
$$

from which we can see that threshold $P_{0}$ in constraint (1) provides a design parameter that allows us to control the collision probability (and so the level of MAC layer contention) on a hop. Importantly, since the channel idle probability $P_{i d l e, h}$ is a observable by all stations on a hop the potential exists to enforce constraint (1) in a decentralized manner, with no need for message passing.

We can regulate $P_{i d l e, h}$ to satisfy constraint (1) by adjusting the station $C W_{\min }$ values to be sufficiently large. Evidently there are many possible combinations of $C W_{\min }$ satisfying the constraint. In particular, this might be achieved by having some stations with very large $C W_{\min }$ and others with small $C W_{\min }$. Such unfair solutions are not of interest in the present context. We borrow from the TCP congestion control literature the use of AIMD to provide a decentralized mechanism for achieving fairness. Specifically, we propose Algorithm 1. 


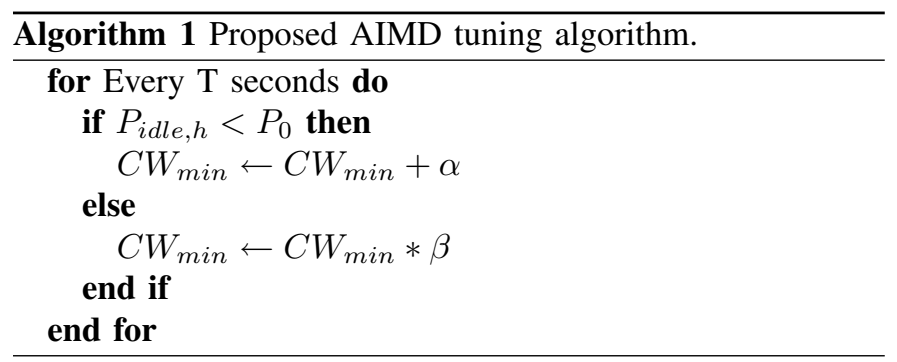

Providing all stations use the same AIMD increase rate $\alpha$ and decrease factor $\beta$, under mild conditions we are guaranteed [22] that stations will, on average, converge to the same value of $C W_{\min }$ while respecting constraint (1) as required.

We briefly comment that the use of channel idle probability has previously been considered in [18] in the context of measuring the link quality in a WLAN. The use of Idle Sense is also considered in [11], [10] in the context of maximizing throughput in a infrastructure mode WLAN. However, this work focusses mainly on optimising throughput in WLANs rather than achieving fairness in mesh networks.

\section{B. Design Parameters}

It can be seen that Algorithm 1 contains four design parameters $\alpha, \beta, P_{0}$ and $T$. We consider these in turn.

The choice of update interval $T$ is determined by the time required to obtain an accurate estimate of the idle probability $P_{\text {idle }, h}$. As a rough guideline, if the measurement noise is roughly white and gaussian we expect that the standard deviation of our $P_{i d l e, h}$ estimate is proportional to $1 / \sqrt{N}$ where $N$ is the number of measurements available. A long update interval $T$ therefore allows more observations and so a more accurate estimate. However, this also slows convergence of Algorithm 1 (see below). In this paper, we use $T=1$ second as a reasonable compromise. We note that this is also a common upper bound of TCP RTT on the Internet and reflects the time-scale over which network conditions are likely to change [28] (currently, WLAN users are mainly using the Internet and the main traffic type is TCP i.e. $80 \%-90 \%$ of traffic is TCP [27]).

From the AIMD analysis in [22], we have that the mean time between backoff events in Algorithm 1 is proportional to $\alpha / T(1-\beta)$. The mean time to converge to the stationary distribution is proportional to $\log 0.05 / \log \beta$ backoff events. We have found values of $\beta=0.75$ (corresponding to a convergence time of roughly 10 backoff events), and $\alpha=4$ to yield good performance across a wide range of network conditions.

The idle probability threshold $P_{0}$ is set to 0.85 initially. In the runtime, $P_{0}$ is tuned with another algorithm. See our technical report for details [5].

\section{Experimental Results}

In this section we use simulations to evaluate the fairness performance achieved when the TXOP adaptation approach of [15] is augmented with the $C W_{\min }$ autotuning algorithm proposed in Section IV-A. This yields a joint TXOP/C $W_{\min }$ tuning algorithm.

We begin by revisiting the example in Section III-B. Fig. 4(a) shows the throughput allocations obtained with the joint $\mathrm{TXOP} / C W_{\min }$ tuning algorithm. It can be seen that all flows, i.e. including both one-hop and two-hop flows, quickly converge to essentially the same goodputs as required. We comment that this is the max-min fair solution for this network topology (defined by an allocation where no flow can achieve higher goodput without reducing the goodput of another flow).

Also shown in Fig. 4(b) is the convergence of the throughput allocations following a change in the network load. Namely, initially the network starts with 5 flows active on each hop and one 2 hop flow. At time 500s an additional 5 flows start at each hop. It can be seen that when the traffic load is increased the network is able to quickly adapt to the new channel state. Fig. 4(c) plots the corresponding $C W_{\min }$ time histories of the wireless stations (to avoid a cluttered plot we only show the $C W_{\min }$ history of a user station that is source for one-hop flows in hop 1, one station in hop 2 and the $C W_{\min }$ history of the user station that is source for the two-hop flow). The action of the $C W_{\min }$ tuning algorithm to increase $C W_{\min }$ when the load increases at time 500 s can be clearly seen. Recall that without this adaptation fairness is lost, see Fig. 3(a).

The topology used in this example (see Fig.2) is of course rather simple (although not necessarily unrealistic). However, the analytic arguments presented at the start of Section IV mean that we can expect the proposed approach to achieve a max-min goodput allocation in general network topologies. We have confirmed this in a wide range of topologies, but due to space restrictions we present results for only one example. Specifically, we consider a network corresponding to a subset of the MIT Roofnet, with topology shown in Fig. IV-C. In this topology, there is an Internet gateway marked as GW. The locations of the user stations and MPs are selected from data received from GPS coordinates of the MIT Roofnet network. There are altogether 21 TCP flows and the allocation of the flows between user stations is detailed in Table. II. There are 13 flows in channel 1,12 in channel 0 , and 8 in channel 2. Channel 1 thus is the bottleneck of this network. If the allocation is max-min fair, flows 0-12 should therefore achieve the same goodput, and similarly flows 13-20 should achieve the same goodput.

Fig. 6(a) shows the goodput allocations achieved using the TXOP-only approach of [15]. It can be seen that the goodput of flow 12 is similar to that of flows 13-20 rather than flows $0-11$, i.e., the allocation is certainly not max-min fair. In comparison, Fig. 6(b) plots the corresponding goodput allocations when the joint $\mathrm{TXOP} / C W_{\min }$ algorithm proposed here is used. It can be seen that max-min fairness is nicely restored.

\section{Channel Noise}

The foregoing analysis considers the impact of contentionrelated losses on fairness, and proposes a joint TXOP/C $W_{\min }$ approach for achieving fairness. In this section we extend 


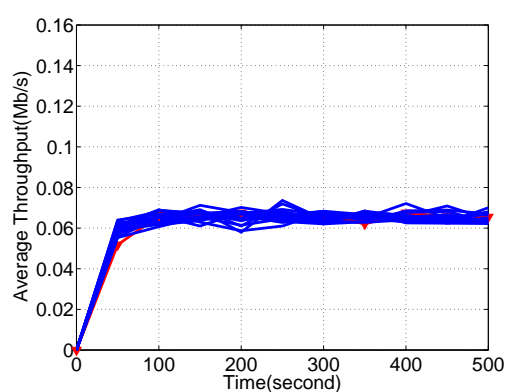

(a) Goodput allocation

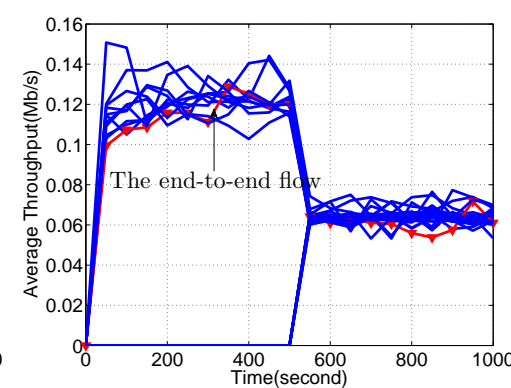

(b) Convergence

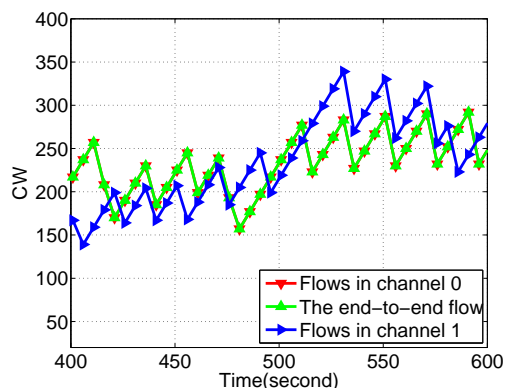

(c) Change of CW in Fig.4(b)

Fig. 4. Goodput allocations when the joint TXOP/C $W_{\min }$ algorithm is used on the topology in Fig. 3.

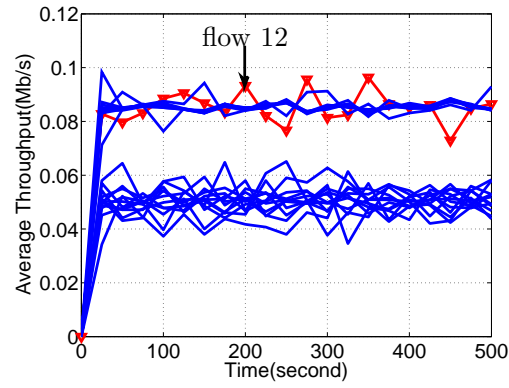

(a) TXOP-only approach

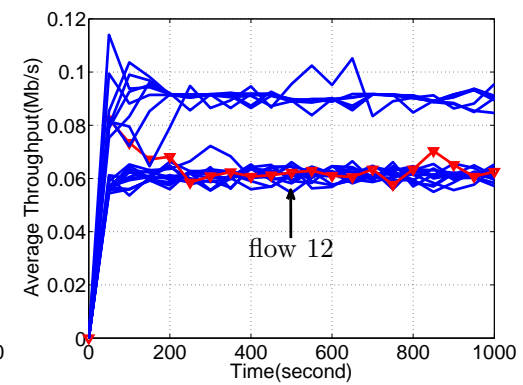

(b) Joint TXOP/CW $W_{\min }$ algorithm

Fig. 6. Goodput allocations in the MIT Roofnet topology

\begin{tabular}{|c|c|c|}
\hline Source station(s) & $\begin{array}{c}\text { Number of flows } \\
\text { on each station }\end{array}$ & Flow ID(s) \\
\hline $0-8$ & 1 & $0-8$ \\
\hline 9 & 3 & $9-11$ \\
\hline 10,11 & 1 & 12,20 \\
\hline 12 & 2 & 18,19 \\
\hline $13-15$ & 1 & $15-17$ \\
\hline 16,17 & 1 & 13,14 \\
\hline
\end{tabular}

TABLE II

Flows IN THE ROOFNET TOPOLOGY IN FIG. IV-C.

consideration to include losses due to channel noise. Due to space restrictions the discussion is, unfortunately, necessarily brief. Nevertheless, we are able to highlight a number of fundamental issues.

Figures 7 and 8 show the goodput allocations in the simple topology of Figure 3 when the TXOP-only and the joint TXOP/C $W_{\min }$ algorithms are used. It can be seen that for a BER of $10^{-} 6$, the noise related losses have little impact on fairness compared to the noise-free case. This is consistent with the recent theoretical results in [23] which establish that the max-min fair allocation varies smoothly as the level of losses is increased. Thus when the loss rate is low it will have only a small impact on fairness.

However, from the results at a BER of $10^{-} 5$ it can be seen that a higher level of channel losses eventually leads to significant unfairness - the two-hop flow now achieves nearly half the goodput of the one-hop flows. Again, this is consistent with [23]. The unfairness arises because the two-hop flow sees a greater end-to-end loss rate than the one-hop flows. In this situation there are several possible solutions. One is to alter the link-level rate control algorithm (that controls the level of FEC and type of PHY modulation) to maintain the BER at a lower level. This seems like a fairly reasonable approach that could be readily implemented (it would involve a trivial extension of our proposed TXOP/C $W_{\min }$ to use time-based rather than packet-based fairness). An alternative is at the first hop to prioritise the two-hop flow to compensate for the channel losses at that hop. However, it seems that this must inevitably involve end-to-end message passing of flow-level information in order to inform the first hop (and later hops in a scenario with more than two hops) of the level of losses experienced by each flow so that the right level of prioritisation can be applied. Consequently, this approach seems less attractive than the linklayer rate control approach, assuming the latter is feasible.

Finally, we note that we might interpret losses at each hop as a "cost" for using that hop. The end-to-end cost is then dependent on the number of hops traversed and this leads naturally to consideration of proportional rather than max-min fairness. However, this is out of scope for the present paper and we leave consideration of proportional rather than maxmin fairness to a future paper.

\section{REFERENCES}

[1] Part 11: wireless LAN medium access control (MAC) and physical layer (PHY) specifications: Medium Access Control (MAC) Quality of Service (QoS) Enhancements, IEEE 802.11e/D8.0, February 2004. 


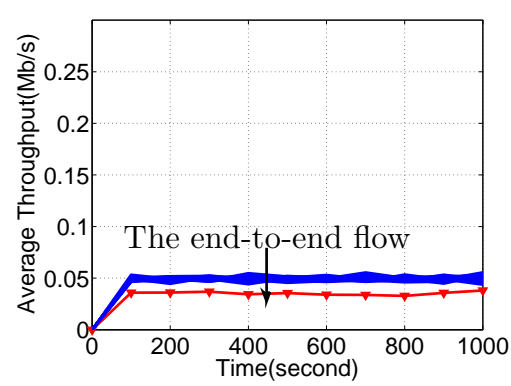

(a) TXOP only, $\mathrm{BER}=10^{-6}$

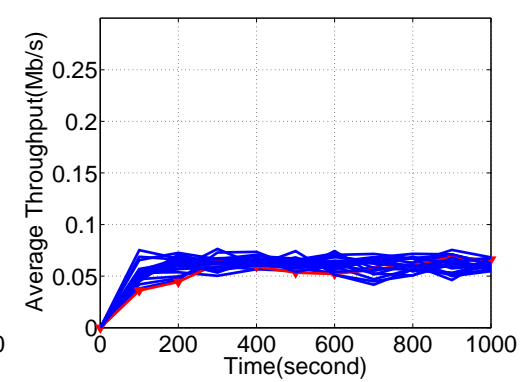

(b) Joint TXOP/CW $W_{\min }$ algorithm, $\mathrm{BER}=10^{-6}$

Fig. 7. Goodput allocation when BER is $10^{-6}$.

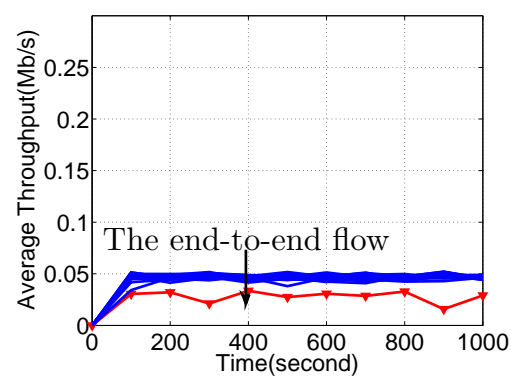

(a) TXOP only, BER $=10^{-5}$



(b) Joint TXOP/CW $W_{\min }$ algorithm, $\mathrm{BER}=10^{-5}$

Fig. 8. Goodput allocation when BER is $10^{-5}$.

[2] V. Badarla, D. Malone, and D. Leith, "Implementing TCP Flow-Level Fairness Using 802.11e in a Multi-Radio Mesh Testbed," IEEE Communications Letters, vol. 12, no. 4, pp. 262-264, Apr. 2008.

[3] P. Bahl, R. Chandra, and J. Dunagan, "SSCH: Slotted seeded channel hopping for capacity improvement in ieee 802.11 adhoc wireless networks," in Proc. ACM MOBICOM, Philadelphia, Sep. 2004, pp. 216-230.

[4] M. Bottigliengo, C. Casetti, C. F. Chiasserini, and M. Meo, "Short-term Fairness for TCP Flows in 802.11b WLANs," in Proc. of IEEE INFOCOM, Mar. 2004, pp. 1383-1392.

[5] Q. Cao, T. Li, and D. J. Leith, "Achieving Fairness in Lossy 802.11e Wireless Multi-Hop Mesh Networks," Technical report 2009, Hamilton Institute.

[6] D. De Couto, D. Aguayo, J. Bicket, and R. Morris, "A high-throughput path metric for multi-hop wireless routing," in Proc. of ACM MobiCom, Sep. 2003, pp. 134-146.

[7] R. Draves, J. Padhye, and B. Zill, "Comparison of routing metrics for static multi-hop wireless networks," in Proc. of ACM SIGCOMM, Aug. 2004.

[8] K. Duffy, D. Leith, T. Li, and D. Malone, "Modeling 802.11 Mesh Networks," IEEE Communication Letters, vol. 10, no. 8, pp. 635-637, Aug. 2006.

[9] V. Gambiroza, B. Sadeghi, and E. W. Knightly, "End to End Performance and Fairness in Multihop Wireless Backhaul Networks," in Proc. of ACM MOBICOM, Sep. 2004

[10] Y. Grunenberger, M. Heusse, F. Rousseau, and A. Duba, "Experience with an Implementation of the Idle Sense Wireless Access Method," in Proc. of ACM CoNext, 2007.

[11] M. Heusse, F. Rousseau, R. Guillier, and A. Duba, "Idle Sense: An Optimal Access Method for High Throughput and Fairness in Rate Diverse Wireless LANs," in Proc. of ACM SIGCOMM, 2005, pp. 121-132.

[12] B.Radunovic, J.Y.Le Boudec, "A unified framework for max-min and min-max fairness with applications”, IEEE/ACM Transactions on Networking, 15(5), pp1073-1083, Oct 2007.

[13] D. Leith, P. Clifford, D. Malone, and A. Ng, "TCP Fairness in 802.11e WLANs," IEEE Communications Letters, vol. 9, no. 11, pp. 964-966, Jun. 2005.
[14] D. Leith and P. Clifford, "A Self-Managed Distributed Channel Selection Algorithm for WLANs," in ACM/IEEE RAWNET, Apr. 2006.

[15] T. Li, D. Leith, D. Malone, and V, Badarla, "Achieving End-to-end Fairness in 802.11e Based Wireless Multi-hop Mesh Networks", Chinacom 2008.

[16] R. Maheshwari, H. Gupta, S. R. Das, "Multichannel MAC Protocols for Wireless Networks," in Proc. IEEE SECON. Reston, VA, Sep. 2006, vol. 2, pp. 393-401.

[17] D. Malone, K. Duffy, and D. Leith, "Modeling the 802.11 Distributed Coordination Function in Nonsaturated Heterogeneous Conditions," IEEE/ACM Transcactions on Networking, vol. 15, no. 1, pp. 159172, Feb. 2007.

[18] D. Malone, P. Clifford, and D. Leith, "MAC Layer Channel Quality Measurement in 802.11," IEEE Communications Letters, vol. 11, no. 2, pp. 143-145, Feb. 2007.

[19] K. Ramachandran, E. Belding-Royer, K. Almeroth, and M. Buddhikot, "Interference-Aware Channel Assignment in Multi-Radio Wireless Mesh Networks," in Proc. of IEEE INFOCOM, Apr. 2006.

[20] B. Raman, "Channel Allocation in 802.11-based Mesh Networks," in Proc. of IEEE INFOCOM, Apr. 2006.

[21] A. Raniwala, P. De, S. Sharma, R. Krishnan, and Tzi-cker Chiueh, "Endto-End Flow Fairness over IEEE 802.11-based Wireless Mesh Networks," in Proc. of IEEE INFOCOM Mini-Symposium, May 2007.

[22] R.N. Shorten, F. Wirth, D.J. Leith, "A positive systems model of TCPlike congestion control: Asymptotic results". IEEE/ACM Transactions on Networking, 14(3), pp616-629, June 2006.

[23] V. G. Subramanian, K. R. Duffy and D. J. Leith, "Existence and uniqueness of fair rate allocations in lossy wireless networks", IEEE Transactions on Wireless Communications, in press.

[24] D. Tang and M. Baker, "Analysis of A Local-Area Wireless Network," in Proc. of ACM MobiCom, Aug. 2000.

[25] I. Tinnirello and S. Choi, "Temporal Fairness Provisioning in Multi-Rate Contention-Based 802.11e WLANs," in Proc. of IEEE WOWMOM, Jun. 2005.

[26] H. Wu, F. Yang, K. Tan, J. Chen, Q. Zhang, and Z. Zhang, "Distributed Channel Assignment and Routing in Multi-radio Multi-channel Multi-hop 




Fig. 5. A subset of the MIT Roofnet topology

Wireless Networks," Journal on Selected Areas in Communications, vol.24, pp. 1972-1983, Nov. 2006

[27] D. Tang and M. Baker, "Analysis of A Local-Area Wireless Network," in Proc. of ACM MobiCom, Aug. 2000.

[28] Z.Zhao,S.Darbha, and A.L.N.Reddy, "A Method for Estimating the Proportion of Nonresponsive Traffic At a Router," in IEEE/ACM Transaction on Networking, vol. 12,no. 4, pp. 708-718, Aug. 2004 